\title{
Expanding roles of NUCB2/nesfatin-1 in neuroendocrine regulation
}

\author{
David García-Galiano ${ }^{1,2}$, Victor M Navarro ${ }^{1,4}$, Francisco Gaytan ${ }^{1,2}$ \\ and Manuel Tena-Sempere ${ }^{1,2,3}$ \\ ${ }^{1}$ Department of Cell Biology, Physiology and Immunology, Faculty of Medicine, University of Córdoba, Avda. Menéndez Pidal s/n, 14004 Córdoba, Spain \\ ${ }^{2}$ CIBER Fisiopatología de la Obesidad y Nutrición, Instituto de Salud Carlos III, 14004 Córdoba, Spain \\ ${ }^{3}$ Instituto Maimónides de Investigaciones Biomédicas (IMIBIC), 14004 Córdoba, Spain \\ ${ }^{4}$ Department of Physiology and Biophysics, University of Washington, Seattle, Washington 98185, USA \\ (Correspondence should be addressed to M Tena-Sempere at Department of Cell Biology, Physiology and Immunology, Faculty of Medicine, \\ University of Córdoba; Email: fi1tesem@uco.es)
}

\begin{abstract}
Nesfatin-1 was originally identified as a hypothalamic neuropeptide, derived from the precursor NEFA (for DNA binding/EF-hand/acidic protein)/nucleobindin 2 (NUCB2), with the ability to suppress food intake, acting in a leptin-independent manner. Departing from this seminal finding, the patterns of expression of NUCB2/nesfatin-1 have been thoroughly characterized in different hypothalamic nuclei and brain areas with proven roles in energy homeostasis, and its potential interactions with other key neuropeptide regulators of appetite have been documented. Intriguingly, recent experimental evidence suggests that NUCB2/nesfatin-1 is also expressed in peripheral tissues with relevant metabolic functions, such as the pancreas, the adipose, and the gut. In addition, evidence is mounting that nesfatin signaling may participate in adaptative responses and in the control of body functions gated by the state of energy reserves, such as puberty onset. Altogether, these observations have broadened our perception of the biological profile of nesfatin-1 that, rather than a simple anorectic signal in the hypothalamus, might operate at different tissues as an integral regulator of energy homeostasis and closely related neuroendocrine functions.
\end{abstract}

Journal of Molecular Endocrinology (2010) 45, 281-290

\section{Identification and structural biology of nesfatin-1}

The hypothalamus is a key integratory area of the brain, where numerous neuropeptides and transmitters are released to participate in the control of essential body functions. Among those, considerable efforts have been devoted recently to unveil the complex circuitries and regulatory factors involved in the central control of food intake and energy balance (Stanley et al. 2005, Sanchez-Lasheras et al. 2010). Unraveling of these pathways and signals is not only physiologically relevant but also poses obvious pharmacological implications, as basis for the rational design of improved therapeutic strategies for the control of body weight, whose disorders (ranging from obesity to anorexia) have grown in prevalence to epidemic proportions in recent years.

In the search for new appetite-controlling signals, Oh $e t$ al. (2006) reported a series of analyses based on a subtraction cloning strategy in SQ-5 cells (derived from lung carcinoma, which expresses leptin and leptin receptors (Tsuchiya et al. 1999)), following stimulation with the PPAR- $\gamma$ ligand, troglitazone. Among the clones identified to be regulated by PPAR- $\gamma$, one was highlighted as i) having a signal peptide (index of secreted proteins), ii) being produced by neuronal- (medulloblastoma HTB185) and adipocyte- (3T3-L1) derived cell lines, and iii) displaying profound stimulated expression following troglitazone (Oh et al. 2006). This target was shown to correspond to the gene encoding nucleobindin 2 (NUCB2), also termed NEFA (for DNA binding/EF-hand/acidic protein). NUCB2 and its closely related NUCB1 are known to be secreted proteins (Miura et al. 1992), but their functions remained largely unknown at that time. Based on the additional observations that NUCB2 is indeed expressed in key hypothalamic nuclei involved in body weight control (see below) and that, when injected into the brain, recombinant NUCB2 protein reduces food intake, this factor was termed nesfatin 
(for NEFA/NUCB2-encoded satiety- and fat-influencing proteins) (Oh et al. 2006).

NUCB2/nesfatin is composed of 396 amino acids, preceded by a 24-amino acid signal peptide (Fig. 1), with very high amino acid sequence homology among rat, mouse, and human species $(>85 \%)$. Structural analyses revealed the presence of several conserved cleavage recognition sites for prohormone convertases (PC) within rat NUCB2/nesfatin sequence, thus suggesting this to be a precursor that gives rise, by differential proteolytic processing, to several active peptides. The predicted (major) fragments of such processing were termed nesfatin-1 (spanning residues 1-82), nesfatin-2 (residues 85-163), and nesfatin-3 (residues 166-396) (Oh et al. 2006). Indeed, by the generation of specific antibodies and implementation of immunohistochemical (IHC) analyses and immunoassays, nesfatin-1 was shown to be produced in the hypothalamus and present in the cerebrospinal fluid (index of this being a secreted neuropeptide). Co-localization of nesfatin-1 and PC3/1 and PC2 suggested that the latter are involved in the cleavage of nesfatin-1 from the NUCB2 precursor (Oh et al. 2006).

\section{Nesfatin-1 as anorectic molecule: biological effects and neuroanatomical distribution}

As indicated above, identification of nesfatin- 1 as a hypothalamic neuropeptide was immediately followed by the characterization of its anorectic effects.

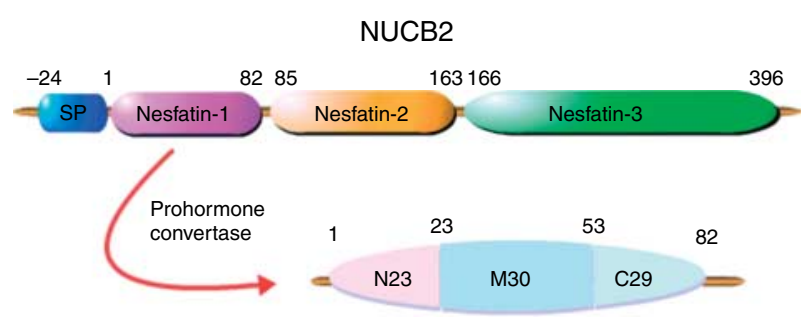

Nesfatin-1

Mid-segment (30 aa): PDTGLYYDEYLKQVIEVLEETDPHFREKLQK

Figure 1 Primary structure of NUCB2 protein, as precursor of nesfatin-1. NUBC2 is composed of 396 amino acids, preceded by a 24-amino acid signal peptide. On the basis of the consensus cleavage sites for prohormone convertases present in its primary sequence, three major peptide products are predicted to be derived from NUBC2: Nesfatin-1 (residues 1-82), nesfatin-2 (residues 85-163), and nesfatin-3 (residues 166-396). In particular, nesfatin-1 seems to be released by the concerted actions of $P C 3 / 1$ and PC2. Functional analyses of three potential fragments of nesfatin-1 molecule (namely, the N-terminal (N23), the C-terminal (C29), and the central (M30) fragments) revealed that the mid-fragment (whose sequence is depicted in the figure) contains the active site for the anorectic effects of nesfatin- 1 . Of note, however, no evidence has been presented to date that such M30 fragment of nesfatin-1 is actually produced endogenously in central or peripheral tissues. SP, signal peptide.
Thus, central (i.c.v.) injection of nesfatin-1 was shown to decrease food intake (and consequently body weight) in rats in a dose-dependent manner. In contrast, neither nesfatin-2 nor nesfatin-3 evoked any anorectic responses (Oh et al. 2006). In good agreement, immunoneutralization of endogenous nesfatin-1, but not of nesfatin-3, significantly enhanced food intake. Likewise, knockdown of hypothalamic NUCB2/nesfatin content by means of a central infusion of an antisense morpholino oligonucleotide (as-MON) consistently increased food intake and body weight in adult rats (Oh et al. 2006). Altogether, these data evidenced that nesfatin-1 is an appetitesuppressive molecule, a contention that has been further confirmed in the rat, where central injection of nesfatin-1 has been shown to decrease dark-phase food intake (Stengel et al. 2009a), and in the mouse, where recombinant human, rat, and mouse nesfatin-1 proteins were capable of acutely suppressing food intake (Shimizu et al. 2009). The above observations have been refined recently by the identification of the active domain of nesfatin-1 molecule causing inhibition of food intake. Thus, based on structural analyses, three different fragments of the nesfatin-1 protein (N-terminal fragment (residues 1-23); mid-fragment (residues 24-53); and C-terminal fragment (residues 54-82); see Fig. 1) were tested for food intake suppression in mice. These analyses revealed that only the mid-fragment was capable to induce anorectic responses, thus unveiling the active core of nesfatin-1 molecule in terms of feeding control (Shimizu et al. 2009). Of important note, however, while the abovementioned fragments were designed on the basis of key features of the predicted secondary structure of nesfatin-1 (such as formation of $\alpha$-helix or secure turn segments of the mature protein), no conclusive demonstration has been presented to date that these fragments are in fact endogenous fragments of nesfatin-1 (Shimizu et al. 2009).

In addition to functional testing, compelling neuroanatomical data support the contention that nesfatin-1 is actually involved in the central pathways controlling food intake. Thus, initial IHC analyses demonstrated the presence of NUCB2/nesfatin-1 protein in several hypothalamic nuclei, such as arcuate nucleus (ARC), paraventricular nucleus (PVN), supraoptic nucleus (SON), lateral hypothalamic area (LHA), and zona incerta, as well as in brainstem areas, such as the nucleus of the solitary tract (nucleus tractus solitarius, NTS), with pivotal roles in regulation of feeding (Oh et al. 2006). These initial observations were later confirmed and extended, with the demonstration of NUCB2/nesfatin-1 mRNA and/or immunoreactivity in the above-mentioned hypothalamic and extrahypothalamic areas (Brailoiu et al. 2007, Foo et al. 2008, Kohno et al. 2008). Of note, such detailed neuroanatomical 
mapping revealed also a wider pattern of central distribution of NUCB2/nesfatin, with detectable expression of its mRNA and/or protein in the periventricular nucleus of the hypothalamus, as well as in a large number of regions outside the hypothalamus, including the Edinger-Westphal nucleus, dorsal motor nucleus of the vagus, caudal raphe nuclei, locus coeruleus, thalamic parafascicular nucleus, and preganglionic sympathetic and parasympathetic neuronal groups, among others (Brailoiu et al. 2007, Foo et al. 2008, Goebel et al. 2009a). Such a wide pattern of distribution of NUCB2/nesfatin-1, with prominent expression not only in hypothalamic nuclei but also in diverse brainstem areas and autonomic centers (Table 1), was already taken as an (indirect) index of its potential function as an integral regulator of energy homeostasis, a contention that has begun to be substantiated recently by, as yet fragmentary, functional evidence that will be summarized in following sections.

\section{Neuroendocrine mechanisms and pathways for the anorectic effects of nesfatin-1}

In addition to the above-mentioned neuroanatomical evidence, functional analyses on the metabolic regulation of NUCB2/nesfatin expression in the

Table 1 Compilation of the major expression and functional data of nucleobindin 2 (NUCB2)/nesfatin-1 system in mammals. For further details and references, see text

\section{Expression}

\section{Central nervous system (CNS)}

Hypothalamus

Paraventricular nucleus (PVN)

Supraoptic nucleus (SON)

Lateral hypothalamic area (LHA)

Zona incerta (ZI)

Tuberal hypothalamic area (THA)

Arcuate nucleus (ARC)

Brainstem and spinal cord

Nucleus tractus solitarius (NTS)

Edinger-Westphal nucleus (EW)

Dorsal motor nucleus of vagus

Caudal raphe nucleus

Thalamic parafascicular nucleus

Locus coeruleus (LC)

Sympathetic and parasympathetic

preganglionic neurons

\section{Peripheral organs/tissues}

Gastrointestinal system

$\mathrm{X} / \mathrm{A}$ cells-gastric glands

Duodenum submucosal layer

Endocrine pancreas- $\beta$ cells

Adipose tissue

\section{Putative function}

Central regulation of feeding behavior and body weight, as anorectic signal acting via leptin-independent and oxytocin/ melanocortin-dependent pathways Central regulation of gastric emptying (forebrain)

Central regulation of stress responses (PVN)

Central regulation of glucose metabolism

Central regulation of arterial pressure

Central autonomic regulation

Central (metabolic) regulation of puberty

Central regulation of feeding behavior and body weight, as anorectic signal acting via leptin-independent and oxytocin/ melanocortin-dependent pathway

Central regulation of stress responses

(EW, NTS, and LC)

Central autonomic regulation

Potential peripheral regulator (anorectic) of food intake in reciprocal interaction with other $\mathrm{X} / \mathrm{A}$ cell product, ghrelin Local regulator of pancreatic function, with potential antihyperglycemic effects

Expression of NUCB2/nesfatin in subcutaneous adipose tissue at much higher levels than in other (visceral) fat depots

\section{Regulation}

Fasting decreases NUCB2/nesfatin-1 expression in the PVN, while (re)feeding activates nesfatin-1 neurons at PVN and SON Cholecystokinin activates nesfatin-1 neurons in the PVN

$\alpha$-MSH increases NUCB2 mRNA levels in the PVN

Stress activates nesfatin-1 neurons in the PVN and SON

Adrenal secretion inhibits NUCB2/nesfatin-1 mRNA at PVN

Co-expression of nesfatin-1 with OT, vasopressin, $\mathrm{MCH}, \mathrm{CART}$, pmTOR, and NPY

Cholecystokinin activates nesfatin-1 neurons in the NTS

Stress activates nesfatin-1 neurons in the NTS, EW, and LC
Fasting decreases gastric expression of NUCB2 mRNA

Fasting nesfatin-1 levels are decreased in patients with type-2 diabetes

High-fat diet increases, whereas fasting decreases, nesfatin-1 protein levels and secretion by the adipose tissue

Inflammatory cytokines, insulin, and dexamethasone enhance nesfatin-1 secretion by subcutaneous adipose tissue There is a positive correlation between body mass index and circulating nesfatin-1 in humans

Unknown 
hypothalamus have further documented its role in the central control of food intake and energy balance. Thus, NUCB2 mRNA levels and nesfatin-1 protein content were shown to be decreased, selectively at the $\mathrm{PVN}$, following $24 \mathrm{~h}$ fasting in adult rats $(\mathrm{Oh}$ et al. 2006), a finding that has been recently confirmed in whole hypothalamic preparations from pubertal female rats (García-Galiano et al. 2010). Conversely, re-feeding potently activated nesfatin-1 neurons at the PVN and $\mathrm{SON}$, as measured by c-Fos immunoreactivity (Kohno et al. 2008). These responses are compatible with the proposed role of nesfatin-1 as an anorectic molecule. Of note, the mechanism whereby nesfatin- 1 conducts such feeding-suppressive actions seems to be independent of leptin signaling, as nesfatin-1 was capable of inhibiting food intake in rodents bearing inactivating mutations of leptin receptor (Oh et al. 2006, Shimizu et al. 2009), and in conditions of leptin resistance, such as dietinduced obesity (Shimizu et al. 2009). In turn, the anorectic effects of leptin were not blocked by immunoneutralization of endogenous nesfatin-1 (Oh et al. 2006). In further support of this leptinindependent mode of action, nesfatin-1 did not appear to be involved in the activation of proopiomelanocortin (POMC) and CART neurons at the ARC, which is one of the primary mechanisms whereby leptin suppresses feeding (Shimizu et al. 2009). Yet, to our knowledge, no report has been published on the direct effects of leptin on the hypothalamic expression of NUCB2/nesfatin-1, or its potential alterations in conditions of leptin deficiency. On the contrary, the anorectic hormone, cholecystokinin, has been shown to activate nesfatin-1 neurons at the PVN and NTS, thus suggesting the involvement of these neuronal populations in mediating the satiating effects of this gut-derived peptide (Noetzel et al. 2009, Stengel et al. 2009a).

Co-expression analyses of NUCB2/nesfatin-1 at specific hypothalamic nuclei, coupled with functional studies, have shed light into the potential neuroendocrine mechanisms and pathways for the anorectic effects of nesfatin-1. Thus, prominent co-localization of NUCB2/nesfatin-1 with oxytocin (OT), and to a lesser extent vasopressin, has been documented at the PVN and SON in rats (Brailoiu et al. 2007, Kohno et al. 2008), and immunoneutralization of endogenous nesfatin-1 reduced OT release at the PVN (Maejima et al. 2009). These observations, together with the fact that OT inhibited food intake in leptin-resistant rats, strongly suggest that nesfatin-induced anorexia involves its ability to activate an oxytocinergic pathway at the PVN (Maejima et al. 2009). The ultimate effector for such a nesfatin-OT circuit might be a population of POMC neurons located at the brainstem NTS, since i) nesfatin-1 and OT were shown to activate POMC neurons specifically at the NTS (Maejima et al. 2009, Shimizu et al. 2009); and ii) the anorectic effects of nesfatin-1 were blocked by an antagonist of the melanocortin-3/4 receptor (Oh et al. 2006). In turn, melanocyte-stimulating hormone $(\alpha-\mathrm{MSH})$ elevated NUCB2 mRNA levels at the PVN (Oh et al. 2006), thus suggesting a bidirectional interaction between these two anorectic systems.

In addition to the OT-melanocortin pathway, other neuronal circuits may be involved as well in mediating the effects of nesfatin-1 on food intake. Thus, nesfatin-1 was found highly co-expressed with the orexigenic peptide, melanin-concentrating hormone $(\mathrm{MCH})$, in neurons at the lateral portion of the tuberal hypothalamic area in rats (Fort et al. 2008); however, the functional relevance of such potential nesfatin-MCH interaction is yet to be evaluated. In addition, electrophysiological studies revealed that nesfatin-1 is capable of inhibiting neuropeptide Y (NPY) neurons at the ARC, as a potential mechanism for its anorectic effects (Price et al. 2008). Finally, it was recently shown that the inhibitory effects of i.c.v. injections of nesfatin-1 on food intake are prevented by the blockade of corticotropin-releasing factor receptor, CRF2, which suggest the involvement of this pathway in mediating the forebrain actions of nesfatin-1 to suppress feeding (Stengel et al. 2009a).

\section{Peripheral nesfatin-1: patterns of expression and putative biological actions}

While nesfatin-1 was initially cataloged as a hypothalamic neuropeptide, growing evidence suggests that, as is the case for many other central regulators of appetite, NUCB2/nesfatin-1 is also expressed in different peripheral tissues with key roles in energy homeostasis and metabolism. Indeed, the original study on the identification of nesfatin-1 already documented the ability of PPAR- $\gamma$ ligands to enhance NUCB2 gene expression in the pre-adipocyte cell line, 3T3-L1 (Oh et al. 2006). Yet, this observation was not immediately followed by the thorough characterization of the expression and potential regulation of NUCB2/nesfatin-1 in the adipose or other metabolic tissues, probably because the appealing roles of nesfatin-1 as a (hypothalamic) neuropeptide controlling food intake at central levels obscured further investigation in this area. Nonetheless, these peripheral facets of NUCB2/nesfatin-1 have recently begun to be characterized; the most salient findings in this front are summarized below.

In keeping with its proven expression in adipocytederived cell lines, the expression of NUCB2 gene and the presence and secretion of nesfatin-1 protein have been recently documented in human and murine adipose tissue (Ramanjaneya et al. 2010). Interestingly, the expression of NUCB2/nesfatin-1 is highly enriched 
in the subcutaneous fat tissue, as compared with other (visceral) depots. In addition, expression and/or secretion of nesfatin-1 in mouse adipose tissue were shown to be significantly increased by diet-induced obesity in vivo, and by inflammatory cytokines, insulin, and dexamethasone in subcutaneous adipose tissue explants ex vivo. In contrast, food deprivation reduced nesfatin-1 protein levels. In good agreement, nesfatin-1 levels markedly increased during differentiation of 3T3-L1 pre-adipocytes into mature adipose cells in vitro (Ramanjaneya et al. 2010). To our knowledge, confirmation of the ability of PPAR- $\gamma$ to enhance NUCB2/nesfatin-1 expression in the adipose tissue has not been reported to date; yet, recent evidence obtained in vitro suggests that such stimulatory action is probably due to stabilization of NUCB2 mRNA, rather than transcriptional activation of the gene, through an ERK1/2 pathway (Yamada et al. 2010). In any event, despite the demonstration of the precisely regulated expression of NUCB2/nesfatin-1 in the adipose tissue, the physiological (or eventual pathophysiological) implications of this phenomenon remain to be characterized. In this front, however, it is worth noting that circulating levels of nesfatin-1 were positively correlated with body mass index in humans (Ramanjaneya et al. 2010). Given the proven ability of nesfatin-1 to cross the blood-brain barrier (Pan et al. 2007, Price et al. 2007) and to induce anorectic responses after peripheral (pharmacological) administration in rodents (Shimizu et al. 2009), it is tempting to explore whether the adipose-borne nesfatin-1 may represent an additional hormonal signal controlling food intake, in a leptin-independent manner.

In addition to its adipose location, evidence is mounting that NUCB2/nesfatin is also expressed in endocrine cells of the gut and the pancreas. On the former, NUCB2/nesfatin-1 immunoreactivity has been demonstrated in the middle and lower segments of gastric mucosal glands, as well as in the submucosal layer of the duodenum in rats (Zhang et al. 2010). Moreover, expression analyses in purified small endocrine cells of gastric mucosa revealed that NUCB2 mRNA levels at this site are higher than in the brain (Stengel et al. 2009b). Interestingly, expression of nesfatin-1 in the midportion of gastric glands co-localized with the orexigenic hormone, ghrelin, presumably in X/A-like cells (Stengel et al. 2009b, $2010 b$ ). In addition, few cells in the base of gastric glands were shown to co-express nesfatin-1 and somatostatin (Stengel et al. 2009b). The observation that X/A-like cells express both ghrelin (orexigenic signal) and nesfatin-1 (anorexigenic factor) is tremendously appealing and suggestive of a central role of this cell type in the hormonal control of food intake. This contention is reinforced by the observation that, contrary to ghrelin, NUCB2 mRNA levels are decreased in small gastric endocrine cells in response to fasting in rats (Stengel et al. 2009b). As further indication of this potential gut-brain pathway, it has been recently shown that abdominal surgery (which induced postoperative ileus) activates nesfatin-1 neurons in key brain nuclei, such as PVN, SON, and NTS (Stengel et al. 2010a), and central injection of nesfatin-1 has been demonstrated to reduce gastric emptying in rats (Stengel et al. 2009a).

Finally, NUCB2/nesfatin-1 immunoreactivity has been recently reported in human and rat islet $\beta$-cells, but not in other cell types of the endocrine pancreas (Foo et al. 2010). In terms of functional relevance, glucose challenge induced a modest but detectable elevation of nesfatin-1 release from rat islets in vitro (Foo et al. 2010), whereas nesfatin-1 administration to $\mathrm{db} / \mathrm{db}$ mice (which are obese and hyperglycemic) lowered circulating glucose levels (Su et al. 2010). Also of interest, mean fasting plasma levels of nesfatin-1 appeared to be decreased in patients with type-2 diabetes ( $\mathrm{Li}$ et al. 2010). Notwithstanding these appealing observations, it has to be stressed also that the magnitude of nesfatin-1 secretory responses to glucose in vitro was eightfold lower than those of insulin (Foo et al. 2010), and that circulating nesfatin-1 does not increase postprandially (e.g. following glucose ingestion) in healthy humans ( $\mathrm{Li}$ et al. 2010), neither is it overtly changed in rodent models of type-2 diabetes (Foo et al. 2010). Altogether, these observations tone down its potential relevance in the control of pancreatic function (or its eventual pathophysiological alterations), and are suggestive of local, rather than endocrine, functions of pancreas-derived nesfatin-1 in the regulation of metabolism.

\section{Nesfatin-1 and the neuroendocrine control of the reproductive axis}

Compelling experimental evidence has demonstrated that central pathways and peripheral signals responsible for body weight homeostasis commonly participate also in the control of related functions, which are gated by the metabolic state of the organism (Fernandez-Fernandez et al. 2006, Hill et al. 2008). Among those, it is well known that puberty onset and fertility are sensitive to the magnitude of body fuel (energy) reserves and under the regulation of key appetite-controlling factors, such as leptin (FernandezFernandez et al. 2006, Hill et al. 2008). Our recent data strongly suggest that central nesfatin-1 signaling might be involved as well in the regulation of the timing of puberty in the female rat. Thus, detailed neuroanatomical studies demonstrated that the patterns of hypothalamic expression of NUCB2 mRNA and NUCB2/nesfatin-1 protein in pubertal female rats are grossly coincident with those reported previously in 


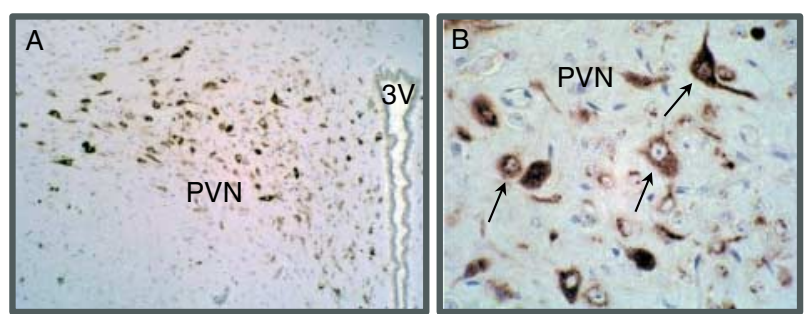

Figure 2 Prominent NUCB2/nesfatin-1 immunoreactivity (IR) in rat hypothalamus. IHC analyses in adult and pubertal rats have documented intense NUCB2 peptide labeling in key hypothalamic nuclei, including the lateral hypothalamic area (LHA), paraventricular nucleus (PVN), zona incerta ( $\mathrm{ZI})$, and supraoptic nucleus (SON), among others. In addition, expression of NUCB2/nesfatin-1 has been demonstrated in a diversity of brain areas outside the hypothalamus. In the figure, detailed images of NUCB2/nesfatin-1 IR at the PVN of a pubertal male rat are shown, at two different magnifications (specific IR denoted by arrows at the highest magnification). IHC analyses were conducted as recently described in García-Galiano et al. (2010), using a specific polyclonal antibody (Phoenix Pharmaceuticals; Ref. H-003-22). $3 \mathrm{~V}$, third ventricle.

adult rodents, with prominent signals in the PVN, LHA, and SON, and fainter expression at ARC (Fig. 2). Of note, hypothalamic NUCB2/nesfatin-1 expression increased during the pubertal transition (i.e. between d-20 (late-infantile) and d-35 (peripubertal)), with a significant heightening of its mRNA levels (as detected by in situ hybridization) at LHA, PVN, and SON, and a threefold increase of its total protein content (as detected by western blot in whole hypothalamic preparations) across this period (García-Galiano et al. 2010). In addition, conditions of negative energy balance, which are linked to disturbed puberty, such as short-term fasting or sustained sub-nutrition, induced a significant decrease in hypothalamic NUCB2 mRNA and protein levels in pubertal females (García-Galiano et al. 2010). Altogether, these expression analyses are suggestive of a precise regional, developmental, and metabolic regulation of NUCB2/nesfatin-1 expression in the hypothalamus at the time of puberty.

Further evidence for the relevance of nesfatin-1 signaling in puberty onset came from functional studies. Thus, i.c.v. administration of nesfatin-1 (at the dose of $50 \mathrm{pmol} / \mathrm{rat}$ ) induced modest but significant elevations of circulating gonadotropins (twofold increase in serum LH levels) in pubertal female rats fed ad libitum. Interestingly, the magnitude of gonadotropin responses to nesfatin-1 was notably increased (ninefold increase in LH levels, at $15 \mathrm{~min}$ after nesfatin-1 injection) in conditions of short-term fasting, in spite of the fact that food deprivation induced a significant decrease in the prevailing serum levels of gonadotropins (García-Galiano et al. 2010). In turn, functional knockdown of endogenous nesfatin-1 tone in the hypothalamus, by means of continuous i.c.v. infusion along pubertal maturation of an as-MON against NUCB2, overtly delayed vaginal opening (an external sign of puberty) and decreased ovarian weights and serum LH levels at the end of the treatment period. Of important note, such a protocol of as-MON infusion evoked a dramatic drop of the hypothalamic levels of NUCB2 protein ( $\sim 20 \%$ of control values), but failed to alter body weight or daily food intake. These observations reinforce the contention that the effects of nesfatin-1 inhibition are genuinely derived from the lowering of its endogenous tone and not indirectly mediated by the potential metabolic alterations linked to as-MON treatment (García-Galiano et al. 2010).

The above findings raise the question of whether, in addition to puberty, nesfatin-1 signaling might participate also in the control of the gonadotropic axis at adulthood. Our initial data, however, failed to demonstrate any detectable gonadotropic responses to i.c.v. injection of nesfatin-1 (50 pmol), neither our protocol of i.c.v. infusion of as-MON against NUCB2 induced overt alterations of the pre-ovulatory surges of LH and FSH in adult, cyclic female rats (García-Galiano et al. 2010). Although these data would suggest a preferential (if not exclusive) action of nesfatin-1 in the control of the gonadotropic axis at puberty, very recent preliminary data suggest that, at higher doses $(1 \mathrm{nmol}$; i.c.v.), nesfatin-1 can elicit LH and FSH responses in adult male rats (Tadross et al. 2010). This would indicate that the gonadotropic axis at adulthood is also responsive (albeit with lower sensitivity) to nesfatin-1; yet, potential sex differences cannot be excluded either. In any event, the biological profile of nesfatin-1, as a central (and peripheral) signal, which is downregulated in conditions of negative energy balance and conducts stimulatory actions in terms of gonadotropin secretion and puberty onset, qualifies this novel factor as a putative metabolic regulator of the hypothalamicpituitary-gonadal axis.

Finally, in the reproductive front, we have obtained preliminary evidence that, as is the case for other central and peripheral regulators of food intake and energy balance, such as ghrelin, resistin, adiponectin, and orexin (Nogueiras et al. 2004, Barreiro et al. 2005, Caminos et al. 2008, Tena-Sempere 2008), NUCB2/ nesfatin-1 is also expressed in the gonads. Thus, we have detected NUCB2 mRNA expression in human, rat, and mouse testis, as well as in the rat ovary (García-Galiano, Gaytan \& Tena-Sempere, manuscript in preparation). In addition, our IHC analyses have revealed prominent NUCB2/nesfatin-1 immunoreactivity in testicular Leydig cells (Fig. 3). Admittedly, many aspects of such gonadal expression of NUCB2/nesfatin-1, such as its (sub)cellular location and putative functional roles in the testis, as well as its developmental, hormonal, and metabolic regulation, await to be elucidated and merit specific investigation. In any event, these 

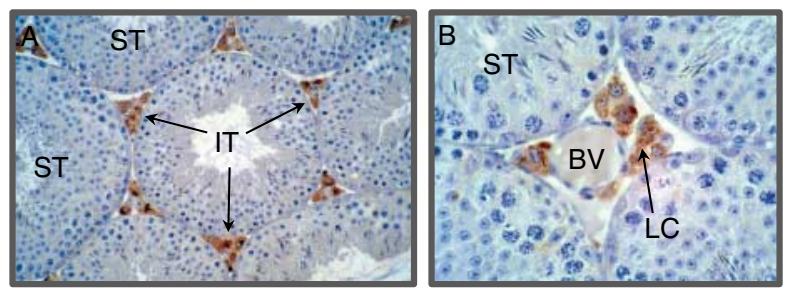

Figure 3 Presence of NUCB2/nesfatin-1 immunoreactivity (IR) in rodent (mouse) testis. IHC analyses in mouse and rat testes have documented intense NUCB2 labeling in interstitial Leydig cells, responsible for testosterone production. In the figure, detailed images of NUCB2/nesfatin-1 IR in testicular sections of an adult mouse are shown, at two different magnifications. Specific nesfatin IR, appearing as brownish cytoplasmic staining in interstitial cells, is denoted by arrows. In contrast, seminiferous tubules were devoid of any discernible IR for nesfatin- 1 . IHC analyses were conducted as recently described by García-Galiano et al. (2010), using a specific polyclonal antibody (Phoenix Pharmaceuticals; Ref. H-003-22). These protein data are in keeping with our recent (as yet unpublished) results on the expression of NUCB2 gene in testes from mouse, rat, and human species. IT, interstitial space; ST, seminiferous tubules; LC, Leydig cells; BV, blood vessel.

emerging data add further complexity to our (as yet incipient) knowledge of the potential sites and mechanisms of action of this molecule in the control of the gonadal axis.

\section{Other neuroendocrine roles of nesfatin-1: adaptative (stress) responses}

In addition to the central control of puberty, compelling evidence is mounting that NUCB2/nesfatin-1 neurons are involved also in the generation of adaptative (stress) responses. Indeed, their wide pattern of distribution in different brain autonomic nuclei (see Table 1) strongly suggested that nesfatin-1 neurons might be recruited upon stress stimuli (Goebel et al. 2009a). Indeed, this contention has been recently confirmed by the demonstration that acute restrain stress is capable of variably activating different populations of NUCB2/nesfatin-1 neurons at the PVN (frequently regarded as the center for the stress axis), SON, and NTS, among other nuclei (Goebel et al. $2009 b$ ). In addition, similar stress protocols induced the activation of a subset of neurons co-expressing nesfatin-1, CART, and urocortin-1 in the non-preganglionic region of the mouse Edinger-Westphal nucleus, which was known to be involved in the response to various stress paradigms (Okere et al. 2010). These observations suggest that nesfatin-1 is part of the repertoire of neuropeptides being activated and effectors for the induction of adaptative responses in conditions of stress.

As further backup to this hypothesis, it has been recently demonstrated that central i.c.v. injection of nesfatin-1 elevated the circulating levels of both ACTH and corticosterone, as major components of the hypothalamic-pituitary-adrenal axis, while bilateral adrenalectomy increased NUCB2 mRNA expression at the PVN (Konczol et al. 2010). These findings demonstrate that central NUCB2/nesfatin participates in the control of the stress axis, and that its expression is under the negative feedback regulation of the major secretory products of the adrenal. Considering the important metabolic component of adaptative responses to stress, such as food intake suppression, it is tenable that nesfatin-1 might participate in the generation of such responses. Moreover, nesfatin-1 signaling in the brain has been also involved in the modulation of cardiovascular responses potentially involved in stress adaptation, such as mean arterial pressure, which is increased upon i.c.v. injection of nesfatin-1 (Yosten \& Samson 2009). As described for its anorectic effects, such hypertensive effects of nesfatin-1 appear to be conducted via a central OT-melanocortin pathway (Yosten \& Samson 2009, 2010).

\section{Nesfatin-1 and neuroendocrine control: open questions and future directions}

Departing from its initial identification as an anorectic hypothalamic neuropeptide (Oh et al. 2006), our perception of nesfatin-1 has significantly enlarged in recent years, with the recognition of its wide pattern of brain distribution, its expression in different peripheral tissues (where nesfatin-1 may represent a secreted molecule), and its ample repertoire of biological functions. While altogether these data are strongly suggestive of a major role of nesfatin-1 as integral regulator of energy homeostasis and related body functions, there are many facets of this signaling system that remain to be solved and are likely to concentrate attention and research efforts in the coming years.

Among those unsolved issues, it is obvious that further progress in this area, both in the physiological and in the pharmacological fronts, will require the identification and molecular characterization of the putative nesfatin receptor(s). Admittedly, some progress has been made in this context, such as the demonstration that nesfatin-1 interacts with a G-proteincoupled receptor (of as yet unknown nature), leading to a rise in intracellular $\mathrm{Ca}^{2+}$ concentrations, by stimulation of $\mathrm{Ca}^{2+}$ influx via N-type, L-type, or $\mathrm{P} / \mathrm{Q}$-type calcium channels, which is linked to PKA activation in specific neuronal populations (Brailoiu et al. 2007, Iwasaki et al. 2009). Yet, it is equally remarkable that cloning of nesfatin receptor(s) has remained elusive to date, a fact that might reflect some specific features of the receptor, e.g. its requirement to heterodimerize with a yet unknown interactive partner(s) that would 
require specific attention. In any event, it is obvious that the precise dissection of the physiological roles and potential pharmacological possibilities of nesfatin-1 (or its analogs) would significantly benefit from the molecular cloning and structure functional analyses of its receptor(s).

In addition, despite the extensive work conducted toward elucidation of the downstream pathways activated upon nesfatin-1 stimulation, our knowledge on the mechanisms and signals responsible for the regulation of NUCB2/nesfatin-1 expression in different tissues (including, prominently, different brain areas) is still incomplete. Efforts in this front will not only allow us to expand our understanding of the individual biological functions of nesfatin-1 in different neuroendocrine axes but will also help to define its actual role as an integral regulator of related body functions, such as energy balance and reproduction. As potentially related with the latter, nesfatin-1 and phosphomammalian target of rapamycin (mTOR) have been recently shown to co-localize in the ARC of male rats (Inhoff et al. 2010), and we have recently demonstrated that mTOR signaling is a novel component of the central pathways linking energy homeostasis and puberty onset (Roa et al. 2009). However, whether these two pathways are functionally related in terms of reproductive control awaits to be evaluated. Similarly, it will be interesting to analyze whether the ability of nesfatin-1 to elicit OT secretion at certain hypothalamic areas, as recently proposed for the control of food intake (Maejima et al. 2009), may also play a role in its reported stimulatory effects on the gonadotropic axis at puberty, since OT has been shown to be a positive regulator of female pubertal maturation (Parent et al. 2008). In the above context, the generation of mouse models of congenital or conditional deficiency of NUCB2/nesfatin-1 would prove also extremely instrumental.

Finally, in addition to increasing physiological recognition, it is reasonable to forecast that in the coming years considerable attention will be drawn by nesfatin-1 in terms of pharmacology and translational medicine. Two features are particularly attractive in this front: first, the ability of systemic administration of nesfatin-1 to suppress food intake (Shimizu et al. 2009); and, secondly, the fact that the anorectic effects of nesfatin-1 are detectable even in conditions of leptin-resistance (Oh et al. 2006, Shimizu et al. 2009). Indeed, recent pharmacological testing has allowed identification of the active core of nesfatin-1 molecule in terms of feeding suppression: the mid-fragment of the 82-amino acid peptide was capable of inhibiting food intake after peripheral injection (Shimizu et al. 2009). This central fragment of 30 amino acids may constitute the basis for the rational design of analogs of nesfatin-1 molecule, with super-agonistic or antagonistic activities and improved pharmacokinetic properties (e.g. pertaining half-life or crossing of blood-brain barrier). Considering that most forms of obesity are linked to variable degrees of leptin resistance, the development of therapeutic strategies for the control of body weight based on the use of nesfatin-1 analogs merits specific investigation. In addition, the facts that nesfatin- 1 is detectable in the circulation in rodent and human species and that its levels have been shown to be altered in different pathological conditions, ranging from diet-induced obesity to type- 2 diabetes, make it appealing to hypothesize that nesfatin-1 may constitute a suitable marker for some metabolic disorders; a possibility that needs to be further substantiated by larger experimental and clinical studies. Altogether, it is anticipated that the research efforts devoted to solve the above basic and clinical questions will help to expose the biology of nesfatin-1, thus allowing to characterize the whole set of effects, mechanisms, and sites of action of this recently identified molecule, which may prove central for the physiological control (and eventual pharmacological manipulation) of energy homeostasis and closely related neuroendocrine functions.

\section{Declaration of interest}

The authors declare that there is no conflict of interest that could be perceived as prejudicing the impartiality of the research reported.

\section{Funding}

The work from the authors' laboratory summarized in this article was supported by grant BFU 2008-00984 (Ministerio de Ciencia e Innovación, Spain), Project P08-CVI-03788 (Junta de Andalucía, Spain), and EU research contract DEER FP7-ENV-2007-1. CIBER is an initiative of Instituto de Salud Carlos III (Ministerio de Sanidad, Spain).

\section{Acknowledgments}

The authors are indebted to the members of the research team at the Physiology Section of the University of Cordoba, who actively participated in the generation of experimental data discussed herein. In addition, the authors are especially grateful to Dr Leonor Pinilla, Enrique Aguilar, and Carlos Dieguez for their helpful comments during preparation of this review.

\section{References}

Barreiro ML, Pineda R, Gaytan F, Archanco M, Burrell MA, Castellano JM, Hakovirta H, Nurmio M, Pinilla L, Aguilar E et al. 2005 Pattern of orexin expression and direct biological actions of orexin-A in rat testis. Endocrinology 146 5164-5175. (doi:10.1210/en.2005-0455)

Brailoiu GC, Dun SL, Brailoiu E, Inan S, Yang J, Chang JK \& Dun NJ 2007 Nesfatin-1: distribution and interaction with a $\mathrm{G}$ proteincoupled receptor in the rat brain. Endocrinology 148 5088-5094. (doi:10.1210/en.2007-0701) 
Caminos JE, Nogueiras R, Gaytan F, Pineda R, Gonzalez CR, Barreiro ML, Castano JP, Malagon MM, Pinilla L, Toppari J et al. 2008 Novel expression and direct effects of adiponectin in the rat testis. Endocrinology 149 3390-3402. (doi:10.1210/en.2007-1582)

Fernandez-Fernandez R, Martini AC, Navarro VM, Castellano JM, Dieguez C, Aguilar E, Pinilla L \& Tena-Sempere M 2006 Novel signals for the integration of energy balance and reproduction. Molecular and Cellular Endocrinology 254-255 127-132. (doi:10.1016/ j.mce.2006.04.026)

Foo KS, Brismar H \& Broberger C 2008 Distribution and neuropeptide coexistence of nucleobindin-2 mRNA/nesfatin-like immunoreactivity in the rat CNS. Neuroscience 156 563-579. (doi:10.1016/ j.neuroscience.2008.07.054)

Foo KS, Brauner H, Ostenson CG \& Broberger C 2010 Nucleobindin-2/nesfatin in the endocrine pancreas: distribution and relationship to glycaemic state. Journal of Endocrinology 204 255-263. (doi:10.1677/JOE-09-0254)

Fort P, Salvert D, Hanriot L, Jego S, Shimizu H, Hashimoto K, Mori M \& Luppi PH 2008 The satiety molecule nesfatin-1 is co-expressed with melanin concentrating hormone in tuberal hypothalamic neurons of the rat. Neuroscience 155 174-181. (doi:10.1016/ j.neuroscience.2008.05.035)

García-Galiano D, Navarro V, Roa J, Ruiz-Pino F, Sánchez-Garrido MA, Pineda R, Castellano JM, Romero M, Aguilar E, Gaytán F et al. 2010 The anorexigenic neuropeptide, Nesfatin-1, is indispensable for normal puberty onset in the female rat. Journal of Neuroscience $\mathbf{3 0}$ 7783-7792. (doi:10.1523/JNEUROSCI.5828-09.2010)

Goebel M, Stengel A, Wang L, Lambrecht NW \& Tache Y $2009 a$ Nesfatin-1 immunoreactivity in rat brain and spinal cord autonomic nuclei. Neuroscience Letters 452 241-246. (doi:10.1016/ j.neulet.2009.01.064)

Goebel M, Stengel A, Wang L \& Tache Y $2009 b$ Restraint stress activates nesfatin-1-immunoreactive brain nuclei in rats. Brain Research 1300 114-124. (doi:10.1016/j.brainres.2009.08.082)

Hill JW, Elmquist JK \& Elias CF 2008 Hypothalamic pathways linking energy balance and reproduction. American Journal of Physiology. Endocrinology and Metabolism 294 E827-E832. (doi:10.1152/ajpendo. 00670.2007)

Inhoff T, Stengel A, Peter L, Goebel M, Tache Y, Bannert N, Wiedenmann B, Klapp BF, Monnikes H \& Kobelt P 2010 Novel insight in distribution of nesfatin-1 and phospho-mTOR in the arcuate nucleus of the hypothalamus of rats. Peptides 31 257-262. (doi:10.1016/j.peptides.2009.11.024)

Iwasaki Y, Nakabayashi H, Kakei M, Shimizu H, Mori M \& Yada T 2009 Nesfatin-1 evokes $\mathrm{Ca}^{2+}$ signaling in isolated vagal afferent neurons via $\mathrm{Ca}^{2+}$ influx through N-type channels. Biochemical and Biophysical Research Communications 390 958-962. (doi:10.1016/ j.bbrc.2009.10.085)

Kohno D, Nakata M, Maejima Y, Shimizu H, Sedbazar U, Yoshida N, Dezaki K, Onaka T, Mori M \& Yada T 2008 Nesfatin-1 neurons in paraventricular and supraoptic nuclei of the rat hypothalamus coexpress oxytocin and vasopressin and are activated by refeeding. Endocrinology 149 1295-1301. (doi:10.1210/en.2007-1276)

Konczol K, Bodnar I, Zelena D, Pinter O, Papp RS, Palkovits M, Nagy GM \& Toth ZE 2010 Nesfatin-1/NUCB2 may participate in the activation of the hypothalamic-pituitary-adrenal axis in rats. Neurochemistry International 57 189-197. (doi:10.1016/j.neuint.2010.04.012)

Li QC, Wang HY, Chen X, Guan HZ \& Jiang ZY 2010 Fasting plasma levels of nesfatin- 1 in patients with type 1 and type 2 diabetes mellitus and the nutrient-related fluctuation of nesfatin-1 level in normal humans. Regulatory Peptides 159 72-77. (doi:10.1016/ j.regpep.2009.11.003)

Maejima Y, Sedbazar U, Suyama S, Kohno D, Onaka T, Takano E, Yoshida N, Koike M, Uchiyama Y, Fujiwara K et al. 2009 Nesfatin-1-regulated oxytocinergic signaling in the paraventricular nucleus causes anorexia through a leptin-independent melanocortin pathway. Cell Metabolism 10 355-365. (doi:10.1016/ j.cmet.2009.09.002)
Miura K, Titani K, Kurosawa Y \& Kanai Y 1992 Molecular cloning of nucleobindin, a novel DNA-binding protein that contains both a signal peptide and a leucine zipper structure. Biochemical and Biophysical Research Communications 187 375-380. (doi:10.1016/ S0006-291X(05)81503-7)

Noetzel S, Stengel A, Inhoff T, Goebel M, Wisser AS, Bannert N, Wiedenmann B, Klapp BF, Tache Y, Monnikes H et al. 2009 CCK-8S activates c-Fos in a dose-dependent manner in nesfatin-1 immunoreactive neurons in the paraventricular nucleus of the hypothalamus and in the nucleus of the solitary tract of the brainstem. Regulatory Peptides 157 84-91. (doi:10.1016/j.regpep. 2009.06.009)

Nogueiras R, Barreiro ML, Caminos JE, Gaytan F, Suominen JS, Navarro VM, Casanueva FF, Aguilar E, Toppari J, Dieguez C et al. 2004 Novel expression of resistin in rat testis: functional role and regulation by nutritional status and hormonal factors. Journal of Cell Science 117 3247-3257. (doi:10.1242/jcs.01196)

Oh IS, Shimizu H, Satoh T, Okada S, Adachi S, Inoue K, Eguchi H, Yamamoto M, Imaki T, Hashimoto K et al. 2006 Identification of nesfatin-1 as a satiety molecule in the hypothalamus. Nature 443 709-712. (doi:10.1038/nature05162)

Okere B, Xu L, Roubos EW, Sonetti D \& Kozicz T 2010 Restraint stress alters the secretory activity of neurons co-expressing urocortin-1, cocaine- and amphetamine-regulated transcript peptide and nesfatin-1 in the mouse Edinger-Westphal nucleus. Brain Research 1317 92-99. (doi:10.1016/j.brainres.2009.12.053)

Pan W, Hsuchou H \& Kastin AJ 2007 Nesfatin-1 crosses the blood-brain barrier without saturation. Peptides 28 2223-2228. (doi:10.1016/j.peptides.2007.09.005)

Parent AS, Rasier G, Matagne V, Lomniczi A, Lebrethon MC, Gerard A, Ojeda SR \& Bourguignon JP 2008 Oxytocin facilitates female sexual maturation through a glia-to-neuron signaling pathway. Endocrinology 149 1358-1365. (doi:10.1210/en.2007-1054)

Price TO, Samson WK, Niehoff ML \& Banks WA 2007 Permeability of the blood-brain barrier to a novel satiety molecule nesfatin-1. Peptides 28 2372-2381. (doi:10.1016/j.peptides.2007.10.008)

Price CJ, Samson WK \& Ferguson AV 2008 Nesfatin-1 inhibits NPY neurons in the arcuate nucleus. Brain Research 1230 99-106. (doi:10.1016/j.brainres.2008.06.084)

Ramanjaneya M, Chen J, Brown JE, Tripathi G, Hallschmid M, Patel S, Kern W, Hillhouse EW, Lehnert H, Tan BK et al. 2010 Identification of nesfatin-1 in human and murine adipose tissue: a novel depotspecific adipokine with increased levels in obesity. Endocrinology 151 3169-3180. (doi:10.1210/en.2009-1358)

Roa J, Garcia-Galiano D, Varela L, Sanchez-Garrido MA, Pineda R, Castellano JM, Ruiz-Pino F, Romero M, Aguilar E, Lopez M et al. 2009 The mammalian target of rapamycin as novel central regulator of puberty onset via modulation of hypothalamic Kiss1 system. Endocrinology 150 5016-5026. (doi:10.1210/ en.2009-0096)

Sanchez-Lasheras C, Konner AC \& Bruning JC 2010 Integrative neurobiology of energy homeostasis-neurocircuits, signals and mediators. Frontiers in Neuroendocrinology 31 4-15. (doi:10.1016/ j.yfrne.2009.08.002)

Shimizu H, Oh IS, Hashimoto K, Nakata M, Yamamoto S, Yoshida N, Eguchi H, Kato I, Inoue K, Satoh T et al. 2009 Peripheral administration of nesfatin-1 reduces food intake in mice: the leptinindependent mechanism. Endocrinology 150 662-671. (doi:10.1210/ en.2008-0598)

Stanley S, Wynne K, McGowan B \& Bloom S 2005 Hormonal regulation of food intake. Physiological Reviews 85 1131-1158. (doi:10.1152/physrev.00015.2004)

Stengel A, Goebel M, Wang L, Rivier J, Kobelt P, Monnikes H, Lambrecht NW \& Tache Y $2009 a$ Central nesfatin-1 reduces dark-phase food intake and gastric emptying in rats: differential role of corticotropin-releasing factor2 receptor. Endocrinology 150 4911-4919. (doi:10.1210/en.2009-0578) 
Stengel A, Goebel M, Yakubov I, Wang L, Witcher D, Coskun T, Tache Y, Sachs G \& Lambrecht NW 2009b Identification and characterization of nesfatin-1 immunoreactivity in endocrine cell types of the rat gastric oxyntic mucosa. Endocrinology 150 232-238. (doi:10.1210/en.2008-0747)

Stengel A, Goebel M, Wang L \& Tache Y $2010 a$ Abdominal surgery activates nesfatin-1 immunoreactive brain nuclei in rats. Peptides 31 263-270. (doi:10.1016/j.peptides.2009.11.015)

Stengel A, Goebel M, Wang L \& Tache Y $2010 b$ Ghrelin, des-acyl ghrelin and nesfatin-1 in gastric X/A-like cells: role as regulators of food intake and body weight. Peptides 31 357-369. (doi:10.1016/ j.peptides.2009.11.019)

Su Y, Zhang J, Tang Y, Bi F \& Liu JN 2010 The novel function of nesfatin-1: anti-hyperglycemia. Biochemical and Biophysical Research Communications 391 1039-1042. (doi:10.1016/j.bbrc.2009.12.014)

Tadross JA, Patterson M, Wynne KJ, Patel S, Suzuki K, Ghatei MA \& Bloom SR 2010 Nesfatin suppresses feeding and stimulates the hypothalamo-pituitary-gonadal axis. Endocrine Journal 57 (Supplement 2) S355-S648. Abstract P3-1-3.

Tena-Sempere M 2008 Ghrelin as a pleotrophic modulator of gonadal function and reproduction. Nature Clinical Practice. Endocrinology and Metabolism 4 666-674. (doi:10.1038/ncpendmet1003)

Tsuchiya T, Shimizu H, Horie T \& Mori M 1999 Expression of leptin receptor in lung: leptin as a growth factor. European Journal of Pharmacology 365 273-279. (doi:10.1016/S0014-2999(98)00884-X)
Yamada M, Horiguchi K, Umezawa R, Hashimoto K, Satoh T, Ozawa A, Shibusawa N, Monden T, Okada S, Shimizu H et al. 2010 Troglitazone, a ligand of peroxisome proliferator-activated receptor-\{gamma\}, stabilizes NUCB2 (Nesfatin) mRNA by activating the ERK1/2 pathway: isolation and characterization of the human NUCB2 gene. Endocrinology 151 2492-2503. (doi:10. 1210/en.2009-1169)

Yosten GL \& Samson WK 2009 Nesfatin-1 exerts cardiovascular actions in brain: possible interaction with the central melanocortin system. American Journal of Physiology. Regulatory, Integrative and Comparative Physiology 297 R330-R336. (doi:10.1152/ajpregu.90867.2008)

Yosten GL \& Samson WK 2010 The anorexigenic and hypertensive effects of nesfatin-1 are reversed by pretreatment with an oxytocin receptor antagonist. American Journal of Physiology. Regulatory, Integrative and Comparative Physiology 298 R1642-R1647. (doi:10. 1152/ajpregu.00804.2009)

Zhang AQ, Li XL, Jiang CY, Lin L, Shi RH, Chen JD \& Oomura Y 2010 Expression of nesfatin-1/NUCB2 in rodent digestive system. World Journal of Gastroenterology 16 1735-1741. (doi:10.3748/ wjg.v16.i14.1735)

Received in final form 5 July 2010

Accepted 3 August 2010

Made available online as an Accepted Preprint 3 August 2010 